Running head: Sensory sensitivity, Intolerance of Uncertainty and Anxiety

\title{
Effects of sensory sensitivity and intolerance of uncertainty on anxiety in mothers of children with autism spectrum disorder
}

\author{
Mirko Uljarevic $^{1}$, Sarah Carrington ${ }^{1}$ and Susan Leekam ${ }^{1}$
}

(1) Cardiff University: Wales Autism Research Centre, School of Psychology, Cardiff University, 70 Park Place, Cardiff, Wales UK, CF10 3A.

Uljarević, M., Carrington, S., \& Leekam, S. (2015). Brief Report: Effects of Sensory Sensitivity and Intolerance of Uncertainty on Anxiety in Mothers of Children with Autism Spectrum Disorder. Journal of Autism and Developmental Disorders, 1-5. $9^{\text {th }}$ August 2015

DOI 10.1007/s10803-015-2557-8, 
Running head: Sensory sensitivity, Intolerance of Uncertainty and Anxiety

\begin{abstract}
This study examined the relations between anxiety and individual characteristics of sensory sensitivity (SS) and intolerance of uncertainty (IU) in mothers of children with ASD. The mothers of 50 children completed the Hospital Anxiety and Depression Scale, the Highly Sensitive Person Scale and the IU Scale. Anxiety was associated with both SS and IU and IU was also associated with SS. Mediation analyses showed direct effects between anxiety and both IU and SS but a significant indirect effect was found only in the model in which IU mediated between SS. This is the first study to characterize the nature of the IU and SS interrelation in predicting levels of anxiety.
\end{abstract}

Keywords: sensory sensitivity, intolerance of uncertainty, parents, anxiety, autism spectrum disorders

Corresponding author: Dr. Mirko Uljarević, Olga Tennison Autism Research Centre, School of Psychological Science, La Trobe University, Victoria 3086, Australia. Email:

M.Uljarevic@1atrobe.edu.au 
Running head: Sensory sensitivity, Intolerance of Uncertainty and Anxiety

\section{Introduction}

Parents of children with developmental disabilities face considerable challenges in their everyday lives and are known to experience elevated levels of stress and anxiety (HamlynWright, Draghi-Lorenz, \& Ellis, 2007; Singer, Ethridge, \& Aldana, 2007). Parents of children with Autism Spectrum Disorder (ASD) are particularly vulnerable to anxiety (Bolton, Pickles, Murphy, \& Rutter, 1998; Kuusikko-Gauffin et al., 2013; Mazefsky, Folstein, \& Lainhart, 2008). Such anxiety may be linked to the stress of managing their child's ASD symptoms (Beck, Hastings, Daley, \& Stevenson, 2004; Bishop, Richler, Cain, \& Lord, 2007; Lecavalier, Leone, \& Wiltz, 2006), but it is also likely to be influenced by individual characteristics in the parent. Identifying the influence of individual parent factors is important given that anxiety has negative implications for interpersonal relations. In this study we focus on two key individual traits that are associated with anxiety in the general population; intolerance of uncertainty and sensory sensitivity (Aron, Aron, \& Jagiellowicz, 2012; McEvoy \& Mahoney, 2011).

Intolerance of uncertainty (IU) is defined as a "relatively broad construct representing cognitive, emotional, and behavioral reactions to uncertainty in everyday life situations" (Freeston, Rhéaume, Letarte, Dugas, \& Ladouceur, 1994). Individuals high on the IU trait have a bias to interpret a novel or unpredictable event as threatening (Freeston et al., 1994; Ladouceur, Gosselin, \& Dugas, 2000). IU was initially considered to be a specific risk factor for the development of Generalized Anxiety Disorder (GAD; Dugas \& Koerner, 2005). More recently it has also been associated with symptoms of other types of anxiety (Carleton, Collimore, \& Asmundson, 2010), depression (Boelen \& Reijntjes, 2009), and obsessive-compulsive disorder

(OCD; Holaway, Heimberg, \& Coles, 2006; Oglesby, Medley, Norr, Capron, Korte, \& Schmidt, 
Running head: Sensory sensitivity, Intolerance of Uncertainty and Anxiety

2013). Furthermore, in the general population, it has been found that IU is a stronger predictor of symptoms of anxiety and depression than risk factors such as neuroticism, anxiety sensitivity, worry and negative affectivity (Carleton et al., 2010; Dugas, Gosselin, \& Ladouceur, 2001; Dugas et al., 2007).

Sensory processing sensitivity (SS) is another important factor contributing to the development and maintenance of affective disorders (Ahadi \& Basharpoor, 2010; Aron \& Aron, 1997; Benham, 2006; Goldsmith, Van Hulle, Arneson, Schreiber, \& Gernsbacher, 2006; Neal, Edelmann, \& Glachan, 2002). SS is a trait that predisposes individuals to be more sensitive to various stimuli from the social and physical environment (Aron et al., 2012). It has been proposed that SS reflects increased functioning of the behavioural inhibition system (BIS), and that individuals high in this trait show increased emotional, biological and stress reactivity to environmental stimulation (Aron et al., 2012).

While both phenomena have been separately associated with anxiety in the general population, there has been virtually no evidence on the potential inter-relationship between SS and IU in predicting anxiety. Only one study has investigated the effect of these phenomena together (Wigham, Rodgers, South, McConachie, \& Freeston, 2015). This study focused on the effect of sensory symptoms, IU and anxiety in predicting repetitive behaviours in children with ASD. The results showed a significant, indirect path from sensory symptoms that predicted repetitive behaviours through IU and anxiety. The main aim of the current study was to further characterize the nature of the specific SS-IU-anxiety inter-relationship to determine whether the same pattern was evident in a sample of adults. We selected a sample of parents of children with autism spectrum disorders (ASD), not only because they are a group at high risk for anxiety, but also 
Running head: Sensory sensitivity, Intolerance of Uncertainty and Anxiety

because new evidence shows that sensory processing problems are also prevalent in this parentgroup, even if they themselves do not have a diagnosis of ASD (Uljarević, Prior \& Leekam, 2014). The presence of IU and SS may further increase the risk for anxiety in this group of parents as elevated IU and sensitivity to environmental stimuli, may lead to the perception of many sources of threat and, therefore, the experience of anxiety symptoms. Establishing the relationship between IU, SS and anxiety has the potential to eventually lead to the design of efficient support programmes for parents and families.

Given previous research in general population samples, it is predicted that both IU and SS would have a direct effect on anxiety. However, IU and SS might also have an indirect effect on anxiety according to two models. First, the presence of high IU may lead to the perception of novel and unpredictable sensory stimuli as threatening and thereby create anxiety; that is, the relation between IU and anxiety may be mediated by SS (model 1). Second, the presence of high sensitivity and a susceptibility to being overwhelmed by environmental stimulation, may lead to an experience of the world as highly unpredictable. Such increased unpredictability could make even the most simple situations and events uncertain and difficult, thereby provoking anxiety. In other words, the relation between SS and anxiety may be mediated by IU (model 2). The second aim of this paper was to explore these two alternative possibilities.

\section{Method}

\section{Participants}

Fifty mothers of children and adolescents with ASD (mean age of children= 10 years 7 months (10.7), $\mathrm{SD}=3.10$; mean age of mothers $=44.4 ; \mathrm{SD}=6.3$ ) living in South Wales, UK, participated 
Running head: Sensory sensitivity, Intolerance of Uncertainty and Anxiety

in this study. Although both fathers and mothers were contacted and encouraged to participate responses were received from mothers only. None of the mothers had a diagnosis of ASD. Parents were asked about both their educational level, number of children and marital status.

All children had an assessment of special needs and had been diagnosed with ASD by a community multidisciplinary team assessment according to DSM-IV-TR (American Psychiatric Association, 2000) and ICD-10 (World Health Organization, 1993) criteria.

\section{Procedures and measures}

Most mothers completed and returned the questionnaires by post, with $16 \%$ completing them as part of a visit to the university. The study was approved by the Cardiff University School of Psychology Research Ethics Committee and all parents have provided informed consent.

The Hospital Anxiety and Depression Scale (HADS; Zigmond \& Snaith, 1983) is a normreferenced questionnaire used to assess anxiety. It contains 14 self-report items, with 7 items forming the Anxiety subscale (HADS-A) used here and 7 items forming the Depression subscale (HADS-D).

The Intolerance of Uncertainty Scale-12 (IU; Carleton, Norton, \& Asmundson, 2007) is a 12item short version of the original 27-item Intolerance of Uncertainty Scale (Freeston, et al., 1994) designed to measure responses to uncertainty.

The Highly Sensitive Person scale (HSP; Aron \& Aron, 1997) is a 27-item scale that provides a detailed assessment of sensory sensitivity (SS) to a wide range of different stimuli and situations. It is one of the most widely-used measures for exploring the relationship between sensory 
Running head: Sensory sensitivity, Intolerance of Uncertainty and Anxiety

sensitivity and affective symptoms in general and clinical populations (see Aron et al., 2012 for a recent review).

The HADS Anxiety scale, the Intolerance of Uncertainty (IU) scale and the Highly Sensitive Person (HSP) scale all had very high internal consistency (Cronbach's $\alpha=.86 ; .91$ and .90 respectively) in a sample of 50 mothers of children with ASD from this paper. There were no missing data and Shapiro-Wilk test indicated that HADS anxiety, IU and HSP scores were normally distributed $(\mathrm{p}=.912 ; 088$; and .078 respectively).

\section{Results}

Data on educational level showed that $32 \%$ of mothers had postgraduate, $30 \%$ had undergraduate or vocational, and $25 \%$ had no post-school qualifications $(12.3 \%$ declined to provide information). Seventy six percent of mothers were married and $24 \%$ divorced. Sixty percent of mothers had only one child and $40 \%$ had more than one child.

Preliminary analysis of demographic factors showed no statistically significant differences in anxiety levels between mothers who were married and divorced $(\mathrm{M}=11.05(\mathrm{SD}=4.63)$ vs $\mathrm{M}=$ $10.08(\mathrm{SD}=2.78) ; \mathrm{t}(48)=.684, \mathrm{p}=.497$; Cohen's $\mathrm{d}=.25)$, mothers who had one child compared with those who had two or more children $(\mathrm{M}=10.23(\mathrm{SD}=4.87)$ vs. $\mathrm{M}=11.70(\mathrm{SD}=3.76)$; $\mathrm{t}(48)=1.19, \mathrm{p}=.236$; Cohen's $\mathrm{d}=.34$ ), nor between mothers who had postgraduate qualifications, undergraduate or vocational qualifications, and mothers who did not have post-school qualifications $(\mathrm{M}=10.35(\mathrm{SD}=2.57)$ vs $\mathrm{M}=10.94(\mathrm{SD}=4.08)$ vs $\mathrm{M}=13.70(\mathrm{SD}=4.60) ; \mathrm{F}$ (2, $42)=2.723, \mathrm{p}=.077)$. 
Running head: Sensory sensitivity, Intolerance of Uncertainty and Anxiety

Anxiety was associated with both SS $(r=.42, p=.003)$ and IU $(r=.498, p<.001)$; IU was also associated with SS $(r=.58 ; \mathrm{p}<.001)$. In order to characterize the nature of this interrelationship, two mediation analyses were performed using the serial mediation model in PROCESS (Hayes, 2012). This method establishes that: (1) there is an effect to be mediated i.e. the independent variable (IV) significantly predicts the dependent variable DV and (2) that the indirect effect defined as the product of the IV $\rightarrow$ MV and the MV $\rightarrow$ DV is statistically significant in the direction predicted by the mediation hypothesis (Preacher \& Hayes, 2004).

The first meditation analysis tested model 1, where SS mediated the relationship between IU and anxiety (Figure 1). Anxiety was predicted by the IU score $\left(R^{2}=.248, \mathrm{t}=3.98 p<.001, \beta=.498\right)$ satisfying the first requirement described above. PROCESS (Hayes, 2012) was used to calculate the significance of the indirect effect. The indirect effect was not significant $(\mathrm{z}=1.2016, \mathrm{p}=.23)$ and in the bootstrapped effect with 5000 resamples, the $95 \%$ confidence interval around the mediated effect was -.01 to .13 , which was also not significant as the range contains zero. This model, therefore, does not satisfy the second requirement described above.

The second mediation analysis tested model 2, where IU mediated the relationship between SS and anxiety (Figure 2). Anxiety was predicted by the SS score $\left(R^{2}=.174, \mathrm{t}=3.18, p=.003, \beta=\right.$ .417). The indirect effect was also significant $(\mathrm{z}=2.21, \mathrm{p}=.02)$ and in the bootstrapped effect with 5000 resamples, the $95 \%$ confidence interval around the mediated effect was between .01 and .07 , which was also significant as the range did not contain zero. 
Running head: Sensory sensitivity, Intolerance of Uncertainty and Anxiety

The main aim of this study was to explore how intolerance of uncertainty (IU) and sensory sensitivity (SS) relate to each other in contributing to anxiety. Correlational analysis showed a three-way inter-relationship between SS, IU and anxiety. Two mediation models were then tested: the first model suggested that SS mediates the relation between IU and anxiety, and the second suggested that IU mediated the relation between SS and anxiety. It is important to highlight the fact that while both IU and SS have been separately associated with anxiety in the general population, there has been no evidence on their potential inter-relationship in predicting anxiety. Therefore, these two mediation hypotheses were exploratory in nature.

Our findings supported the second mediation model. In other words, the presence of high sensitivity and a susceptibility to being overwhelmed by environmental stimulation may lead to an experience of the world as highly unpredictable. Such increased unpredictability could make even the most simple situations and events uncertain and difficult, thereby provoking anxiety.

In addition to contributing to the literature on anxiety in the general population by characterizing the inter-relation between SS, IU and anxiety, this study contributes to the literature on anxiety in parents of children with ASD. The finding that IU was associated with anxiety is in line with previous findings suggesting that IU is one of the key contributing factors in the development, maintenance, and exacerbation of affective problems in the general population (Carleton et al., 2010) and in children and adolescents with ASD (Boulter, Freeston, South, \& Rodgers, 2014; Wigham et al., 2015). It is possible that those parents with high IU perceive more sources of threat during stressful events and, therefore, experience greater anxiety than parents who better tolerate and manage uncertainty. Support for this hypothesis comes from two studies demonstrating that the presence of IU discriminated between individuals who developed anxiety 
Running head: Sensory sensitivity, Intolerance of Uncertainty and Anxiety

and those who did not while experiencing the same levels of stress in their everyday lives (Chen \& Hong, 2010; Ciarrochi, Said, \& Deane, 2005).

Consistent with previous findings in both the general (Aron et al., 2012) and ASD population (Lane, Reynolds, \& Dumenci, 2012; Lidstone, Uljarević et al., 2014), anxiety in mothers was highly associated with SS. Individuals with high SS may be easily overwhelmed in response to the demands of their environment and novel situations, and behave in a cautious manner in order to constrain and control their environment as far as possible, akin to the temperamental feature of behavioural inhibition (Aron et al., 2012).

This study had a number of limitations. First, this study is limited by the relatively small sample size and by the use of a self-report questionnaire. Second, we were only able to recruit mothers of children with ASD. Despite the acknowledged difficulties in engaging fathers in research of this kind (Wilson \& Prior, 2011), inclusion of fathers and looking at the family system in future research is important (Karst \& Van Hecke, 2012). Third, although none of the mothers that took part in this study had a diagnosis of ASD, it will be important for future research to explore the potential contribution of the broader autism phenotype (Piven, Palmer, Jacobi, Childress, \& Arndt, 1997), and a range of other parent and family factors that might influence these effects. We found no evidence for a statistically significant influence of marital status, education level or number of children on mothers' levels of anxiety. However, we did not explore the quality of the marriage nor the level of support that mothers receive from their spouses. Previous studies have shown that parents' perception of the level of support that they receive is a significant contributor to parents' stress and anxiety level (Boyd, 2002). It will be important to explore in future studies how perceived and actual levels of support contribute to the inter-relationship 
Running head: Sensory sensitivity, Intolerance of Uncertainty and Anxiety

between sensory sensitivity, IU and anxiety. Finally, employing a longitudinal design and exploring how children's characteristics interact with parental IU and sensory sensitivity in future studies would be of great value in determining true mediation.

This work has potential clinical implications, as the finding of the association of IU, SS and anxiety in mothers of children with ASD could inform the development of evidence-based services designed to support parents' needs. For example, the Unified Protocol for the Transdiagnostic Treatment of Emotional Disorders (Barlow, Allen, \& Choate, 2004) incorporates elements from several evidence-based treatments and was designed to be applied to anxiety and other problem areas with strong emotional components. This approach has been found to reduce both intolerance of uncertainty and symptoms of anxiety and depression in groups of patients diagnosed with heterogeneous anxiety disorders (Boswell, ThompsonHollands, Farchione, \& Barlow, 2013).

\section{Summary}

This is the first study to characterize the interrelationship between sensory sensitivity (SS), intolerance of uncertainty (IU) and anxiety. Furthermore, this is the first study to demonstrate the contribution of IU and SS to the levels of anxiety in mothers of children and adolescents with ASD. Future work should include replication of these results with a larger sample, the use of other informants, and the use of carefully designed experimental protocols to disentangle the neurophysiological mechanisms underlying SS, IU and their relationship to anxiety. 
Running head: Sensory sensitivity, Intolerance of Uncertainty and Anxiety

\section{References}

Ahadi, B., \& Basharpoor, S. (2010). Relationship Between Sensory Processing Sensitivity, Personality Dimensions and Mental Health. Journal of Applied Sciences, 1-5.

American Psychiatric Association (2000). Diagnostic and statistical manual of mental disorders ( $4^{\text {th }}$ ed., text rev.). Washington, DC: Author.

Aron, E., \& Aron, A. (1997).Sensory-processing sensitivity and its relation to introversion and emotionality. Journal of Personality and Social Psychology, 73, 345-368.

Aron, E., Aron A., \& Jagiellowicz, J. (2012) Sensory processing sensitivity: A review in the light of the evolution of biological responsivity. Personality and Social Psychology Review, $16,262-282$.

Barlow, D. H., Allen, L. B., \& Choate, M. L. (2004). Towards a unified treatment for emotional disorders. Behavior Therapy, 35(2), 205-230.

Beck, A., Hastings, R. P., Daley, D., \& Stevenson, J. (2004). Pro-social behaviour and behaviour problems independently predict maternal stress. Journal of Intellectual and Developmental Disability, 29(4), 339-349.

Benham, G. (2006). The highly sensitive person: Stress and physical symptom reports. Personality and Individual Differences, 40(7), 1433-1440.

Bishop, S. L., Richler, J., Cain, A. C., \& Lord, C. (2007). Predictors of perceived negative impact in mothers of children with autism spectrum disorder. American Journal on Mental Retardation, 112(6), 450-461. 
Running head: Sensory sensitivity, Intolerance of Uncertainty and Anxiety

Boelen, P. A., \& Reijntjes, A. (2009). Intolerance of uncertainty and social anxiety. Journal of Anxiety Disorders, 23,130-135.

Bolton, P. F., Pickles, A., Murphy, M., \& Rutter M. (1998). Autism, affective and other psychiatric disorders: Patterns of familial aggregation. Psychological Medicine. 28, 385395.

Boswell, J. F., Thompson-Hollands, J., Farchione, T. J., \& Barlow, D. H. (2013). Intolerance of uncertainty: a common factor in the treatment of emotional disorders. Journal of Clinical Psychology, 69(9), 630-45.

Boulter, C., Freeston, M., South, M., \& Rodgers, J. (2014). Intolerance of uncertainty as a framework for understanding anxiety in children and adolescents with autism spectrum disorders. Journal of Autism and Developmental Disorders, 44(6), 1391-402.

Boyd, B. A. (2002). Examining the relationship between stress and lack of social support in mothers of children with autism. Focus on Autism and Other Developmental Disabilities, 17(4), 208-215.

Carleton, R. N., Norton, M. A., \& Asmundson, G. J. G. (2007). Fearing the unknown: A short version of the intolerance of uncertainty scale. Journal of Anxiety Disorders, 21, 105117.

Carleton, R. N., Collimore, K. C., \& Asmundson, G. J. G. (2010). "It's not just the judgements —it's that I don't know": intolerance of uncertainty as a predictor of social anxiety. Journal of Anxiety Disorders, 24, 189-195. 
Running head: Sensory sensitivity, Intolerance of Uncertainty and Anxiety

Chen, C. Y., \& Hong, R. Y. (2010). Intolerance of uncertainty moderates the relation between negative life events and anxiety. Personality and Individual Differences, 49(1), 49-53.

Ciarrochi, J., Said, T., \& Deane, F. P. (2005). When simplifying life is not so bad: The link between rigidity, stressful life events, and mental health in an undergraduate population. British Journal of Guidance \& Counselling, 33(2), 185-197.

Dugas, M. J., Gosselin, P., \& Ladouceur, R. (2001). Intolerance of uncertainty and worry: investigating specificity in a nonclinical sample. Cognitive Therapy and Research, 25(5), $551-558$.

Dugas, M. J., \& Koerner, N. (2005). The cognitive behavioural treatment of generalized anxiety disorder: Current status and future directions. Journal of Cognitive Psychotherapy, 19(1), 61-81.

Dugas, M. J., Sevard, P., Gaudet, A., Turcotte, J., Laugesen, N., Robichaud, M., et al. (2007). Can the components of a cognitive model predict the severity of generalized anxiety disorder? Behavior Therapy, 38, 169-178.

Freeston, M., Rhéaume, J., Letarte, H., Dugas, M. J., \& Ladouceur, R. (1994). Why do people worry? Personality and Individual Differences, 17(6), 791-802.

Goldsmith, H. H., Van Hulle C. A., Arneson, C. L., Schreiber, J. E., \& Gernsbacher, M. A. (2006). A population-based twin study of parentally reported tactile and auditory defensiveness in young children. Journal of Abnormal Child Psychology, 34, 393-407. 
Running head: Sensory sensitivity, Intolerance of Uncertainty and Anxiety

Hamlyn-Wright, S., Draghi-Lorenz, R., \& Ellis, J. (2007). Locus of control fails to mediate between stress and anxiety and depression in parents of children with a developmental disorder. Autism, 11, 489-501.

Hayes, A. F. (2012). PROCESS: A versatile computational tool for observed variable mediation, moderation, and conditional process modeling [White paper]. Retrieved from http:// www.afhayes.com/public/process2012.pdf.

Holaway, R. M., Heimberg, R. G., \& Coles, M. E. (2006). A comparison of intolerance of uncertainty in analogue obsessive-compulsive disorder and generalized anxiety disorder. Journal of Anxiety Disorders, 20, 158-174.

Karst, J. S., \& Van Hecke, A. V. (2012). Parent and family impact of autism spectrum disorders: a review and proposed model for intervention evaluation. Clinical Child and Family Psychology Review, 15(3),247-277.

Kuusikko-Gauffin, S., Pollock-Wurman, R., Mattila, M. L., Jussila, K., Ebeling, H., Pauls, D., \& Moilanen, I. (2013). Social anxiety in parents of high-functioning children with autism and Asperger Syndrome. Journal of Autism and Developmental Disorders, 43(3), 521-9.

Ladouceur, R., Gosselin, P., \& Dugas, M. J. (2000). Experimental manipulation of intolerance of uncertainty: A study of a theoretical model of worry. Behaviour Research and Therapy, $38,933-941$.

Lane, S. J., Reynolds, S., \& Dumenci, L. (2012). Sensory overresponsivity and anxiety in typically developing children and children with autism and attention deficit hyperactivity 
Running head: Sensory sensitivity, Intolerance of Uncertainty and Anxiety

disorder: cause or coexistence? American Journal of Occupational Therapy, 66, 595603.

Lecavalier, L., Leone, S., \& Wiltz, J. (2006). The impact of behaviour problems on caregiver stress in young people with autism spectrum disorders. Journal of Intellectual Disability Research, 50(3), 172-183.

Lidstone, J., Uljarević, M., Sullivan, J., Rodgers, J., McConachie, H., Freeston, M., et al. (2014). Relations among restricted and repetitive behaviors, anxiety and sensory features in children with autism spectrum disorders. Research in Autism Spectrum Disorders, 8(2), 82-92.

Mazefsky, C. A, Folstein, S. E., \& Lainhart, J. E. (2008). Brief Report: Overrepresentation of mood and anxiety disorders in adults with autism and their first degree relatives: What does it mean? Autism Research, 1. 193-197.

McEvoy, P. M., \& Mahoney, A. E. J. (2011). Achieving certainty about the structure of intolerance of uncertainty in a treatment-seeking sample with anxiety and depression. Journal of Anxiety Disorders, 25, 112-122.

Neal, J. A., Edelmann, R. J., \& Glachan, M. (2002).Behavioural inhibition and symptoms of anxiety and depression: is there a specific relationship with social phobia? British Journal of Clinical Psychology, 41(4), 361-374. 
Running head: Sensory sensitivity, Intolerance of Uncertainty and Anxiety

Oglesby, M. E., Medley, A. N., Norr, A. M., Capron, D. W., Korte, K. J., \& Schmidt, N. B. (2013). Intolerance of uncertainty as a vulnerability factor for hoarding behaviors. Journal of Affective Disorders, 145, 227-231.

Piven, J., Palmer, P., Jacobi, D., Childress, D., \& Arndt, S. (1997). Broader autism phenotype: evidence from a family history study of multiple-incidence autism families. American Journal of Psychiatry, 154, 185-190.

Preacher, K. J., \& Hayes, A. F. (2004). SPSS and SAS procedures for estimating indirect effects in simple Mediation models. Behavior Research Methods, Instruments, \& Computers, $36,717-731$.

Singer, G. H. S., Ethridge, B. L., \& Aldana, S. L. (2007). Primary and secondary effects of parenting and stress management interventions for parents of children with developmental disabilities: A meta-analysis. Mental Retardation and Developmental Disabilities, 13(4), 357-369.

Uljarević, M., Prior, M., \& Leekam, S. R. (2014). First evidence of sensory atypicality in mothers of children with Autism Spectrum Disorder (ASD). Molecular Autism, 5:26.

Wigham, S., Rodgers, J., South, M., McConachie, H., \& Freeston, M. (2015). The Interplay Between Sensory Processing Abnormalities, Intolerance of Uncertainty, Anxiety and Restricted and Repetitive Behaviours in Autism Spectrum Disorder. Journal of Autism and Developmental Disorders, 45(4), 943-952. 
Running head: Sensory sensitivity, Intolerance of Uncertainty and Anxiety

Wilson, K. R., \& Prior, M. R. (2011).Father involvement and child well-being. Journal of Paediatrics and Child Health, 47, 405-407.

World Health Organisation (1993). Manual of the interational statistical classification of diseases and related health problems tenth revision (Vol 1, pp. 375-377). Geneva, Switzerland: Author.

Zigmond, A. S., \& Snaith, R.P. (1983). The Hospital Anxiety And Depression Scale. Acta Psychiatrica Scandinavica, 67, 361-370.

\section{Acknowledgments}

We greatly appreciate the support and time given by the parents who were involved in the research.

\section{Conflict of Interest}

Authors declare no conflict of interest. 
Running head: Sensory sensitivity, Intolerance of Uncertainty and Anxiety

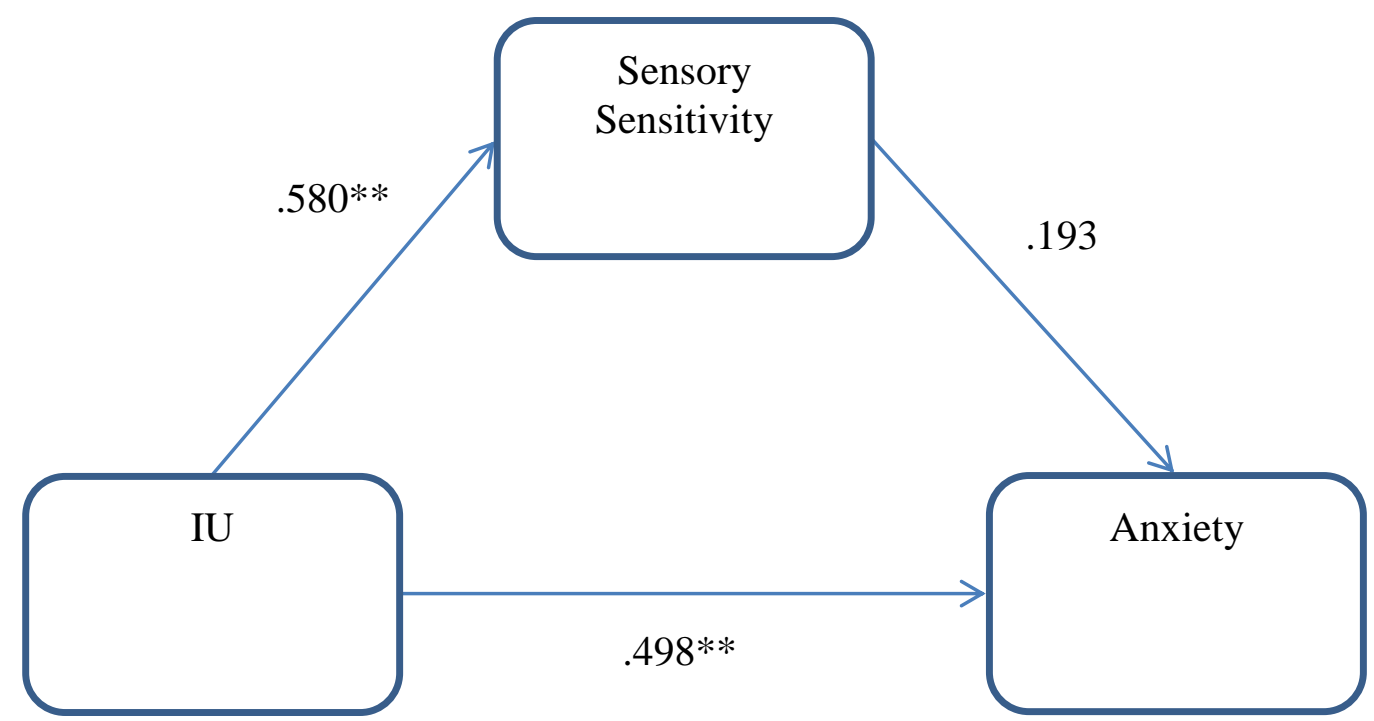

Figure 1.Mediation Model 1. ${ }^{*}=\mathrm{p}<.01 ; * *=\mathrm{p}<.001$ 
Running head: Sensory sensitivity, Intolerance of Uncertainty and Anxiety

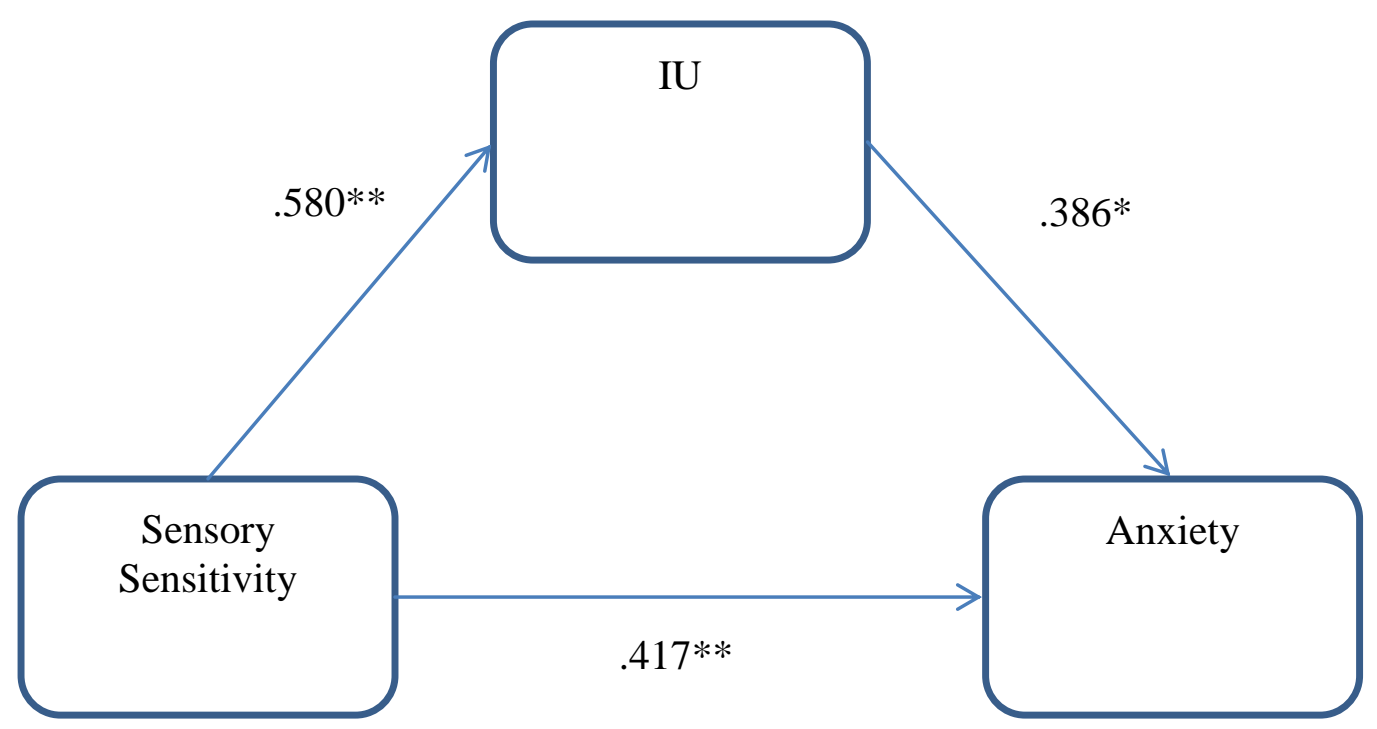

Figure 2.Mediation Model 2. ${ }^{*}=\mathrm{p}<.01 ; * *=\mathrm{p}<.001$ 